\title{
Erratum to: Minimal polar swimmer at low Reynolds number
}

Ankita Pandey and R. Aditi Simha ${ }^{a}$

Department of Physics, Indian Institute of Technology Madras, Chennai 600036, India

Original article: Eur. Phys. J. E (2012) 35: 52, DOI: 10.1140/epje/i2012-12052-7

Received 27 June 2012

Published online: 27 July 2012 - (C) EDP Sciences / Società Italiana di Fisica / Springer-Verlag 2012

The expression for the constant $A$ appearing in eq. (13) and defined in the line below should read $A=\epsilon \delta / 4 \tau$ instead of $A=\epsilon \gamma / 4 \tau$.

\footnotetext{
${ }^{a}$ e-mail: aditi@physics.iitm.ac.in
} 